\title{
GW23-e1143 DUAL-AXIS ROTATIONAL CORONARY ANGIOGRAPHY CAN REDUCE PEAK SKIN DOSE AND SCATTERED DOSE: A PHANTOM STUDY
}

doi:10.1136/heartjnl-2012-302920ad.5

Liu Huiliang, Jin Zhigeng, Yang Shengli, Luo Jianping, Ma Dongxing, Liu Ying, Han Wei, Liu Huiliang. Division of Cardiology, General Hospital of Chinese People's Armed Police Forces, Beijing 100039, China

Objectives to evaluate the peak skin dose received by the patient and scattered dose to the operator during the dual-axis rotational coronary angiography and compared to standard coronary angiography.

Methods An anthropomorphic phantom was used to simulate coronary angiography and generate radiation dose. Procedures included the simulation of standard coronary angiography taking into account the complexity of the individual lesion and dual-axis rotational coronary angiography. The peak skin dose was measured with thermoluminescent dosimeter arrays and scattered dose with a dosimeter at predefined height (approximately at the level of left arm) at the operator's location.

Results Measurements showed, compared to standard coronary angiography, there is a reduction in peak skin dose (the overall reduction: 68\%) and scattered dose (the overall reduction: 58\%) with the use of dual-axis rotational coronary angiography. Moreover, these reductions become greater in the set of complicated lesion examinations (a reduction of $82 \%$ for peak skin dose and $70 \%$ for scattered dose).

Conclusions In comparison to standard coronary angiography, dual-axis rotational coronary angiography can reduce peak skin dose and scattered dose, especially for the complicated lesion examinations. The major reasons seem to be the overall decreased amount of applied energy during the dual-axis rotational coronary angiography, and that it can spread the administered dose over a larger area and minimise the peak skin dose. 